\title{
Smartphone snapshot mapping of skin chromophores under triple- wavelength laser illumination
}

Janis Spigulis

Ilze Oshina

Anna Berzina

Alexander Bykov 


\title{
Smartphone snapshot mapping of skin chromophores under triple-wavelength laser illumination
}

\author{
Janis Spigulis, ${ }^{a, *}$ Ilze Oshina, ${ }^{a}$ Anna Berzina, ${ }^{a}$ and Alexander Bykov ${ }^{b}$ \\ aUniversity of Latvia, Biophotonics Laboratory, Institute of Atomic Physics and Spectroscopy, Riga, Latvia \\ bUniversity of Oulu, Optoelectronics and Measurement Techniques Unit, Faculty of Information Technology and Electrical Engineering, \\ Oulu, Finland
}

\begin{abstract}
Chromophore distribution maps are useful tools for skin malformation severity assessment and for monitoring of skin recovery after burns, surgeries, and other interactions. The chromophore maps can be obtained by processing several spectral images of skin, e.g., captured by hyperspectral or multispectral cameras during seconds or even minutes. To avoid motion artifacts and simplify the procedure, a single-snapshot technique for mapping melanin, oxyhemoglobin, and deoxyhemoglobin of in-vivo skin by a smartphone under simultaneous three-wavelength (448-532-659 $\mathrm{nm}$ ) laser illumination is proposed and examined. Three monochromatic spectral images related to the illumination wavelengths were extracted from the smartphone camera RGB image data set with respect to crosstalk between the RGB detection bands. Spectral images were further processed accordingly to Beer's law in a three chromophore approximation. Photon absorption path lengths in skin at the exploited wavelengths were estimated by means of Monte Carlo simulations. The technique was validated clinically on three kinds of skin lesions: nevi, hemangiomas, and seborrheic keratosis. Design of the developed add-on laser illumination system, image-processing details, and the results of clinical measurements are presented and discussed. @ 2017 Society of Photo-Optical Instrumentation Engineers (SPIE) [DOI: 10.1117/1.JBO.22.9.091508]
\end{abstract}

Keywords: multispectral imaging; skin chromophore mapping; laser illumination design; smartphone applications.

Paper 160816SSR received Nov. 30, 2016; accepted for publication Feb. 10, 2017; published online Mar. 2, 2017.

\section{Introduction}

Abnormal distribution of skin absorbing chromophores (e.g., epidermal melanin and dermal hemoglobin) is a clinical feature typical for burns, bruises, and various kinds of skin malformations, including tumors. ${ }^{1}$ Skin chromophore distribution maps can provide qualitative and quantitative information on the severity of pathology, its development, and/or skin recovery processes. Simple and fast skin chromophore mapping is of special interest for dermatologists, cosmetologists, oncologists, emergency clinicians, surgeons, and forensic experts, as it facilitates decision taking and further strategy development. However, the procedures with commercially available devices that can provide skin chromophore distribution maps (e.g., SIAscope $)^{2}$ are time-consuming, the equipment is expensive and is cable-connected to a computer, which limits its wide applications in personalized medicine and in the field conditions.

Each chromophore has its specific absorption spectrum, ${ }^{3}$ so the remitted light intensity from a spot of skin (containing one or more chromophores) depends on the detection wavelength and composition of the comprised chromophores. A set of several spectral images of the same skin area, captured within different wavelength bands, can be converted into clinically significant parametric images - chromophore distribution maps. ${ }^{4}$ There are two main approaches for obtaining spectral images: by narrowband spectral filtering of the image sensor at spectrally broadband illumination of the target area or by means of spectrally narrowband illumination. The first approach is widely used in hyperspectral imaging (HSI) systems that capture sequential images of the target at several tens of adjacent spectral bands, e.g., using interference filters, ${ }^{5}$ acousto-optical, or liquid crystal filters. ${ }^{6}$ Commercial and custom-designed HSI systems have been successfully used for skin chromophore mapping. ${ }^{5,7-9}$ However, routine clinical applications of the HSI systems are limited due to their robustness, expensiveness, and the need for powerful computing resources to process numerous spectral images (including those eventually not containing useful information). Another advanced chromophoresensitive technology, spatial frequency domain spectroscopy, ${ }^{9}$ faces similar limitations. The HSI image acquisition process may last from several seconds ${ }^{9}$ to a minute or longer, ${ }^{10}$ so movements of the skin target area during the procedure can cause image artifacts. Special image stabilization algorithms ${ }^{11}$ could reduce or fully avoid the motion artifacts, but it takes extra time and computing resources. Additional problems arise if the extinction coefficient of a particular chromophore is notably changing within a single band wavelength interval, typically 10 to $20 \mathrm{~nm}$ or more; in such cases, calculations of complicated integrals comprising spectral features of the chromophore absorbance and the filtered HSI band shape are necessary.

The second approach, spectrally selective illumination, has been mainly used in skin multispectral imaging (MSI) systems where sequentially switchable nonoverlapping spectral bands of illumination (e.g., by several LEDs with different peak wavelengths at typical spectral half-bandwidth $\sim 20$ to $50 \mathrm{~nm}$ ) are exploited for obtaining spectral images. ${ }^{12,13}$ Advanced MSI systems are portable and self-sustained, much cheaper than HSI systems, and better adapted for clinical use. The MSI processing procedure is also faster due to lower number of the spectral images, typically between 3 and 8 . Still, skin motion artifacts 
and processing of relatively broadband spectral images may cause serious problems.

To improve the performance of the MSI chromophore mapping, acquisition time of the spectral images and the spectral bandwidth of illumination have to be minimized. An optimal approach would be the single-snapshot technique dealing with monochromatic (single-wavelength) spectral images. A method for extraction of three monochromatic spectral images from a single snapshot RGB image data set, taken under simultaneous three-wavelength illumination, has been recently proposed ${ }^{14}$ and experimentally validated on color targets. ${ }^{15}$ The previous studies confirmed that separate readings of three RGB detection channels are sufficient to spectrally isolate reflected light at each illumination wavelength, if the crosstalk among those channels is properly taken into account. ${ }^{16}$ A table-top setup comprising RGB board-camera and fiber optic system for simultaneous illumination of target by the 473-, 532-, and 659-nm laser lines has been tested for skin chromophore mapping, as well. ${ }^{17}$ In the results, physiologically plausible chromophore maps of melanin, oxyhemoglobin, and deoxyhemoglobin in pigmented and vascular skin lesions have been obtained by a single snapshot. This laboratory setup, however, was not well-adapted for patient measurements, so new designs of compact/portable equipment for skin chromophore mapping in clinical environment are of essential interest.

Currently, there are about two billion smartphones in use worldwide, ${ }^{18}$ many of them in the pockets of doctors or other clinical personnel. Smartphones comprise high-resolution digital RGB cameras, white LED illuminators, multifunctional displays, and powerful data processors with the ability to transmit the data (including specific images) via mobile networks. This set of features makes smartphones attractive for potential diagnostic applications, e.g., evaluation of human skin condition. Several dermatology-specific mobile applications, including those intended for skin melanoma early detection, have been developed. ${ }^{19-21}$ If aiming at skin chromophore distribution mapping, the spectral information contained in smartphone digital images, taken under the built-in white LED illumination, appears to be insufficient. Narrowband filtering of the white illumination (e.g., by a set of interference filters) ${ }^{22}$ could improve the situation, but more promising from this point seems the use of specially designed small size multispectral add-on illuminators, e.g., based on narrowband color LEDs. ${ }^{23}$

This paper describes implementation of the RGB singlesnapshot approach for the mapping of three main skin chromophores using a smartphone with attached compact three-wavelength laser illuminator. Image processing principles, design of the system, and the first results of its clinical tests are discussed below.

\section{Method}

\subsection{Concept}

The general concept of snapshot skin chromophore mapping at fixed wavelengths is illustrated in Fig. 1. Let us suppose that RGB color image of a skin pathology spot, surrounded by healthy skin, is captured under illumination that comprises only three equal intensity spectral lines at wavelengths $\lambda_{1}, \lambda_{2}$, and $\lambda_{3}$ (the vertical lines in Fig. 1). With respect to the spectral sensitivity of an RGB image sensor and the cross-talk between its detection bands at the particular wavelengths, three monochromatic spectral images are extracted from the color image data

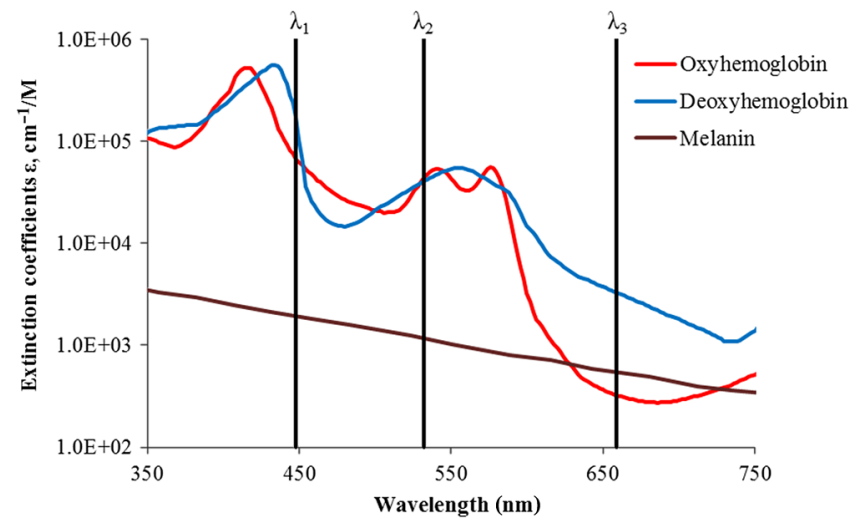

Fig. 1 Absorption of three main skin chromophores at three fixed wavelengths. ${ }^{24,25}$

set by the technique described in Refs. 14-17. The threechromophore skin model ${ }^{26}$ suggests that the dominant absorbers in skin at any of the fixed wavelengths $\lambda_{j}(j=1,2,3)$ are oxyhemoglobin (further abbreviated as a), deoxyhemoglobin (b), and melanin (c) - see the crossings of their absorption curves with the vertical lines in Fig. 1. If the skin surface reflection is suppressed (e.g., by means of crossed polarizers), variations in chromophore composition would lead to changes of the diffusely reflected light intensities at each of the fixed wavelengths. Such variations in the pathology region relative to the healthy skin can be estimated by measuring reflected light intensities from equally sized regions of interest in the pathology $\left(I_{j}\right)$ and the adjacent healthy skin $\left(I_{0 j}\right)$. The ratios $I_{j} / I_{0 j}$ at each pixel or pixel's group of three monochromatic spectral images contain information on the concentration increase or decrease of all three regarded chromophores $\Delta c_{i}(i=a, b, c)$, which can be further mapped over the whole image area.

\subsection{Image Processing}

The above-mentioned intensity changes at three exploited wavelengths due to absorption can be represented in terms of the Beer-Lambert-Bouguer law ${ }^{27}$

$$
\left\{\begin{array}{l}
\ln \left(\frac{I_{1}}{I_{01}}\right)=-l_{1}\left[\Delta c_{a} \cdot \varepsilon_{a}\left(\lambda_{1}\right)+\Delta c_{b} \cdot \varepsilon_{b}\left(\lambda_{1}\right)+\Delta c_{c} \cdot \varepsilon_{c}\left(\lambda_{1}\right)\right] \\
\ln \left(\frac{I_{2}}{I_{02}}\right)=-l_{2}\left[\Delta c_{a} \cdot \varepsilon_{a}\left(\lambda_{2}\right)+\Delta c_{b} \cdot \varepsilon_{b}\left(\lambda_{2}\right)+\Delta c_{c} \cdot \varepsilon_{c}\left(\lambda_{2}\right)\right] \\
\ln \left(\frac{I_{3}}{I_{03}}\right)=-l_{3}\left[\Delta c_{a} \cdot \varepsilon_{a}\left(\lambda_{3}\right)+\Delta c_{b} \cdot \varepsilon_{b}\left(\lambda_{3}\right)+\Delta c_{c} \cdot \varepsilon_{c}\left(\lambda_{3}\right)\right]
\end{array}\right.
$$

where $\varepsilon_{i}\left(\lambda_{j}\right)$ is extinction coefficients of three regarded chromophores at three exploited wavelengths and $l_{j}$ is absorption path length in skin at a particular wavelength. Chromophore concentration increase or decrease at each image pixel (or selected group of pixels) is found by solving the linear equation system (Eq. 1) with abbreviated measured quantities $k_{j}=\ln \left(I_{j} / I_{0 j}\right)$ :

$$
\begin{aligned}
& \Delta c_{a}=A_{1} \cdot k_{1}+A_{2} \cdot k_{2}+A_{3} \cdot k_{3} \\
& \Delta c_{b}=B_{1} \cdot k_{1}+B_{2} \cdot k_{2}+B_{3} \cdot k_{3} . \\
& \Delta c_{c}=C_{1} \cdot k_{1}+C_{2} \cdot k_{2}+C_{3} \cdot k_{3}
\end{aligned}
$$

The coefficients $A_{j}, B_{j}$, and $C_{j}$ comprise numerical values of the corresponding chromophore extinction coefficients $\varepsilon_{i}\left(\lambda_{j}\right)$ 
and absorption path lengths $l_{j}$ at the three exploited wavelengths. The respective extinction coefficients for oxyhemoglobin, deoxyhemoglobin, and melanin for our working wavelengths 448, 532, and $659 \mathrm{~nm}$ are presented in Table 1.

The Beer-Lambert-Bouguer law is originally addressed to absorption of a light beam passing through a slab of substance with thickness $x$. If this law is adapted to the diffusely remitted light geometry, slab thickness $x$ has to be replaced by the mean path length $l_{j}$ of the backscattered photons inside the skin at a particular wavelength. To estimate the $l_{j}$ values with respect to light scattering properties, Monte Carlo (MC) simulations in a frame of the two-layered skin model ${ }^{28}$ (epidermal and dermal layers followed by the infinite subcutaneous tissue layer) were performed. Optical parameters of the tissue layers for the considered wavelengths were averaged from the number of available sources for typical Caucasian skin. ${ }^{29-33}$ See Table 2.

The simulations have been performed for various thicknesses of the epidermal layer $(200,150$, and $100 \mu \mathrm{m})$ and a dermis of $1800 \mu \mathrm{m})$. In the simulations, uniform illumination beam with a diameter of $50 \mathrm{~mm}$ has been accounted. The diffusely backscattered (remitted) photons were collected from the same area accounting for the aperture angle of $10 \mathrm{deg}$. MC simulations were performed using a parallel (message passing interface) MC code for light propagation in biotissue-like media developed and verified in the earlier studies. ${ }^{34-36}$ As the outcome, the distributions of the detected photons over their total path length and maximal penetration depth were generated. The mean path length and the mean penetration depth were calculated by weighted average taking into account the weight of the corresponding photon packets. The simulation results for skin with an epidermal thickness of $200 \mu \mathrm{m}$ are presented in Fig. 2. MC simulations showed increased photon penetration depth and total path length with growing wavelength, the mean path length of the remitted photons being $\sim 4$ to 5 times longer than their average penetration depth. The MC calculation results are presented in Table 3. One can see that the mean path length of photons in skin at a fixed wavelength is quite insensitive $(<10 \%)$ to the twofold increase/decrease of the epidermal thickness. Consequently, different skin epidermal thicknesses should not seriously influence the data obtained by the proposed technique. The clinical images presented in Sec. 3 were processed using the MC-estimated mean path length values for 0.2-mm-thin skin epidermis.

To summarize, our algorithm for image processing includes the following steps:

1. RGB image comprising pathology and adjacent healthy skin is captured under illumination by three discrete wavelengths (disabling all automatic settings of the camera).

Table 1 The extinction coefficients of three main skin chromophores $^{24,25}$ at three exploited wavelengths.

\begin{tabular}{lccc} 
Chromophore & $\begin{array}{l}\varepsilon(448 \mathrm{~nm}) \\
(1 / \mathrm{mol} . \mathrm{cm})\end{array}$ & $\begin{array}{c}\varepsilon(532 \mathrm{~nm}) \\
(1 / \mathrm{mol} . \mathrm{cm})\end{array}$ & $\begin{array}{c}\varepsilon(659 \mathrm{~nm}) \\
(1 / \mathrm{mol} . \mathrm{cm})\end{array}$ \\
\hline Melanin & 1935 & 1170 & 541 \\
Oxyhemoglobin & 67,044 & 45,400 & 320 \\
Deoxyhemoglobin & 173,320 & 41,336 & 3227 \\
\hline
\end{tabular}

Table 2 Optical properties of biotissue layers used in the simulations ( $\lambda$, wavelength, $\mu_{\mathrm{s}}$, scattering coefficient, $\mu_{\mathrm{a}}$, absorption coefficient, $g$, anisotropy factor, $n$, refractive index).

\begin{tabular}{lccccc} 
& $\lambda(\mathrm{nm})$ & $\mu_{\mathrm{s}}\left(\mathrm{mm}^{-1}\right)$ & $\mu_{\mathrm{a}}\left(\mathrm{mm}^{-1}\right)$ & $g$ & $n$ \\
\hline Epidermis & 659 & 20 & 0.20 & 0.90 & 1.36 \\
& 532 & 27 & 0.60 & 0.85 & 1.36 \\
& 448 & 33 & 1.0 & 0.84 & 1.36 \\
& 659 & 18 & 0.12 & 0.90 & 1.40 \\
Dermis & 532 & 22 & 0.30 & 0.85 & 1.40 \\
& 448 & 27 & 0.65 & 0.84 & 1.40 \\
& 659 & 7 & 0.07 & 0.80 & 1.40 \\
Subcutaneous & 532 & 10 & 0.40 & 0.76 & 1.40 \\
tissues & 448 & 14 & 0.70 & 0.75 & 1.40 \\
& & & & &
\end{tabular}

2. Three monochromatic spectral images are extracted from the digital image data set using the RGB crosstalk correction algorithm, ${ }^{14}$ with respect to actual relative intensities of the illumination lines.

3. Equal area regions of interest are selected in the pathology and healthy skin zones of the monochromatic images.

4. Pathology-to-healthy relative spectral reflectances $I_{j} / I_{0 j}$ at the three exploited wavelengths and the values $k_{j}=\ln \left(I_{j} / I_{0 j}\right)$ are calculated for all image pixels or selected pixel's groups.

5. The concentration increase/decrease of three chromophores in the pathology is calculated (2) for each pixel or pixel's group.

6. Distribution maps of three skin chromophores are constructed and displayed.

\subsection{Design of the Illumination Unit}

A compact smartphone-compatible three-wavelength illuminator has been designed, assembled, and tested in laboratory and clinics. The illumination unit comprised six laser modules, an optical element for laser beam management, and an electronics compartment. Figure 3(a) shows the design details and outlook of the operating prototype with a smartphone on it [Fig. 3(b)]. To ensure uniform three-wavelength illumination of around target area, an optical element with a flat ring-shaped laser diffuser was developed. ${ }^{37}$ The illumination wavelengths 448, 532, and $659 \mathrm{~nm}$ were emitted by three pairs of compact $20-\mathrm{mW}$ power laser modules (models PGL-DF-450 nm-20 mW-15011564, PGL-VI-1-532 nm-20 mW-15030443, and PGL-DF-655 nm$20 \mathrm{~mW}-150302232$, Changchun New Industries Optoelectronics Tech. Co., Ltd). Laser modules [1, Fig. 3(a)—shown 3 out of 6] of each equal-wavelength pair were mounted at opposite sides on the internal wall of a hollow three-dimensional (3-D)-printed plastic shielding cylinder (2); the round bottom opening of this 
(a)

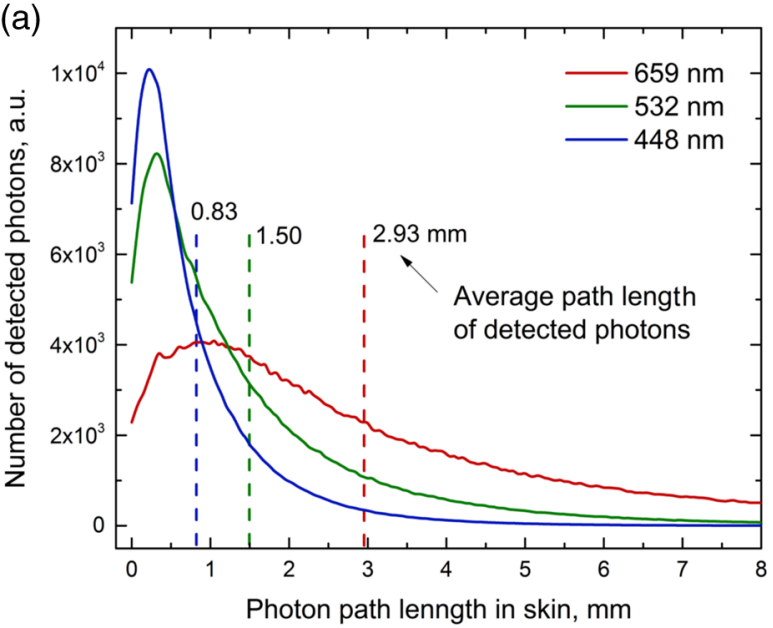

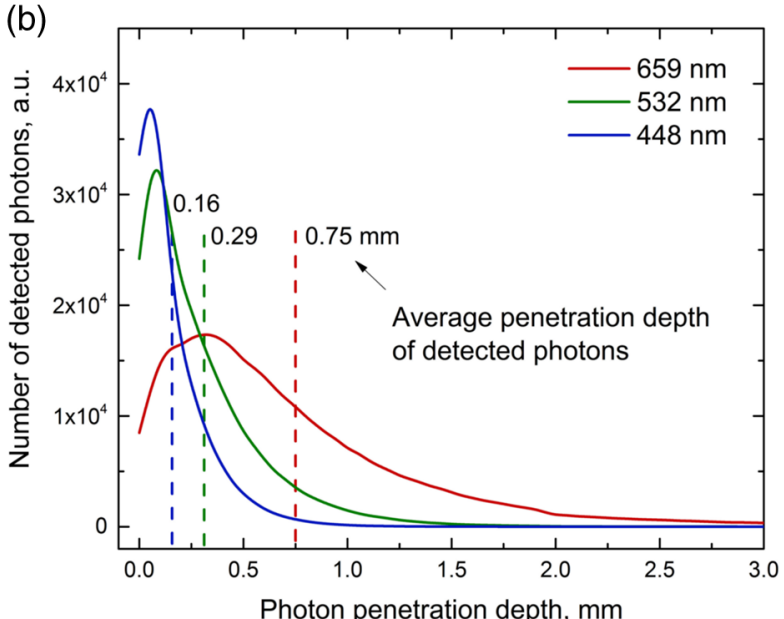

Fig. 2 Monte Carlo simulated distributions of the detected photons over their total path length (a) and penetration depth (b) inside the skin (thickness of epidermis: $200 \mu \mathrm{m}$ ) for three exploited wavelengths. The mean path length and the mean penetration depth were calculated by weighted average taking into account weight of the corresponding photon packets.

Table 3 MC-estimated penetration depths and path length in skin of the remitted photons. Last column: ratio between the mean path length and the mean penetration depth.

\begin{tabular}{lcccc}
$\begin{array}{l}\text { Wavelength } \\
(\mathrm{nm})\end{array}$ & $\begin{array}{c}\text { Epidermal } \\
\text { thickness } \\
(\mathrm{mm})\end{array}$ & $\begin{array}{c}\text { Mean } \\
\text { penetration } \\
\text { depth }(\mathrm{mm})\end{array}$ & $\begin{array}{c}\text { Mean path } \\
\text { length in skin } \\
(\mathrm{mm})\end{array}$ & $\begin{array}{c}\text { The } \\
\text { calculated } \\
\text { ratio factor }\end{array}$ \\
\hline 448 & 0.20 & 0.16 & 0.83 & 5.19 \\
& 0.15 & 0.17 & 0.88 & 5.18 \\
& 0.10 & 0.18 & 0.92 & 5.11 \\
532 & 0.20 & 0.29 & 1.50 & 5.17 \\
& 0.15 & 0.31 & 1.57 & 5.06 \\
& 0.10 & 0.32 & 1.64 & 5.13 \\
659 & 0.20 & 0.75 & 2.93 & 3.91 \\
& 0.15 & 0.76 & 2.95 & 3.88 \\
& 0.10 & 0.76 & 2.98 & 3.92 \\
\hline
\end{tabular}

cylinder (diameter $40 \mathrm{~mm}$ ) was in contact with skin and formed the field of view for the smartphone camera, situated $80 \mathrm{~mm}$ apart. All six coaxial laser beams were pointed to the 45-deg conical reflecting edge of a transparent disc (3) (beam collector) made of standard Plexiglas; after reflections they were turned radially toward the internal ring-shaped flat milky-Plexiglas diffuser (4). The upper and side surfaces of the collector/diffuser unit were mirrored by a vacuum-sputtered Al-coating. As a result, the flat diffuser (4) evenly illuminated the $65-\mathrm{mm}$ distant skin target area simultaneously by three laser wavelengths. More details on the laser beam collector-diffuser are provided in Fig. 4.

The smartphone-model Google Nexus5, 8 Mpx image sensor SONY IMX179 with known RGB-sensitivities ${ }^{38}$-was placed on a flat sticky platform (5) [Fig. 3(a)] with a round window for the smartphone rear camera, coaligned with the internal opening of the diffuser (4). The advantage of flat sticky platform design ${ }^{23,37,39}$ is the ability to fix most models of smartphones or tablet computers, independent of their dimensions; it was implemented by means of a double-sided tape. The round camera window was covered by a film polarizer (high contrast linear polarizing film ${ }^{40}$ ); another film with orthogonal direction of polarization covered the diffuser (4) from the bottom, so avoiding detection of skin surface-reflected light by the smartphone camera. The estimated spatial resolution of the imaging system was better than $0.1 \mathrm{~mm}$.

A block diagram of the three-wavelength illumination device is shown in Fig. 5. Microcontroller (STM32F103) managed operation of the three-laser power modules via control of the laser power supply unit (PSU). Each pair of laser modules could be switched on and off independently by means of the control buttons. Two rechargeable batteries (Samsung 18650 3.7V Li-Ion, 2.8 Ah) and laser power supply circuits were placed in the 3-D-printed plastic compartment (6) below the platform (5). Target illumination intensity was adjusted to ensure linearity of photodetection by the smartphone camera; its automatic settings were switched off by the AZ Camera software, so the R-, G-, and B-outputs of the image sensor responded according to the spectral sensitivities of its three detection bands. 38

The above-described optical design ensured acceptable uniformity of target illumination. It was initially checked at all combinations of illumination wavelengths $(448,532$, and $659 \mathrm{~nm})$ and image sensor detection channels (R, G, B), using white paper as the target. Figure 6 shows the obtained illumination intensity distributions, detected at separated RGB output channels in two cases: (i) when only one wavelength was used for illumination $(659 \mathrm{~nm}$ detected at R-channel, $532 \mathrm{~nm}$ detected at G-channel, and $448 \mathrm{~nm}$ detected at B-channel, the upper row) and (ii) when all three wavelength of illumination were simultaneously switched on (the lower row).

\subsection{Protocol of the Clinical Measurements}

The mobile smartphone-illuminator system passed preliminary clinical case study under approval of the local ethics committee 
(a)

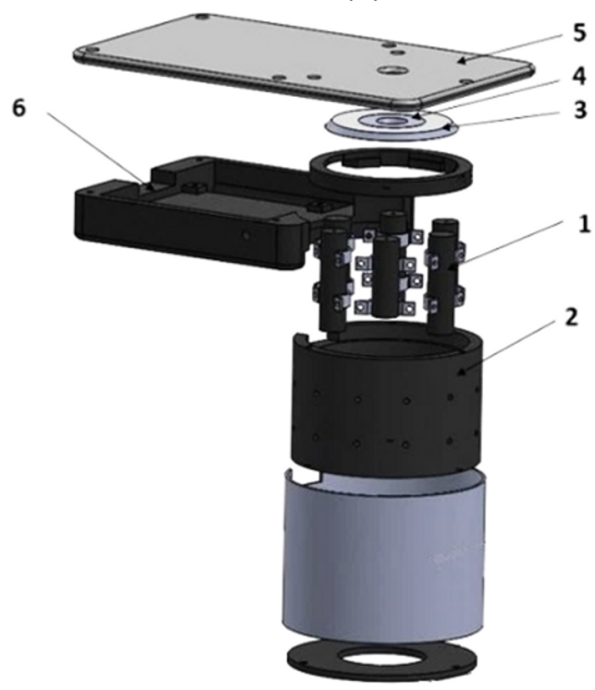

(b)

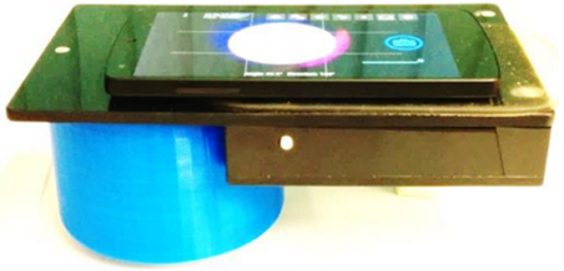

Fig. 3 (a) 3-D model of the three-wavelength illumination unit and (b) the mobile prototype with smartphone on it. (1) Laser modules (three pairs, 448-532-659 nm), (2) shielding cylinder, (3) collector of laser beams, (4) flat ring-shaped diffuser of laser light, (5) sticky platform for the smartphone, (6) electronics compartment.

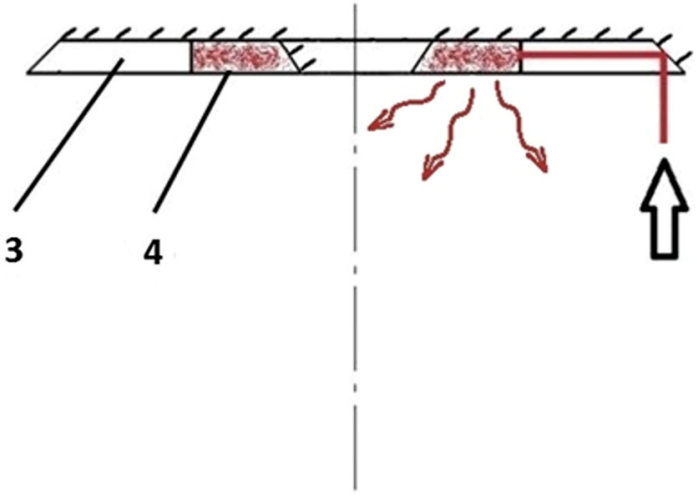

Fig. 4 Optical scheme of the laser beam collecting-diffusing element. ${ }^{36}$ The arrow denotes one of six coaxial laser beams that are turned radial by 45 -deg conical reflecting edge of the transparent disk 3 and then downscattered by means of the internal diffusing ring 4. Upper surfaces of both disks are Al-mirrored. Dimensions: external diameter $50 \mathrm{~mm}$, inner-outer diameters of the diffusing ring 10 to $40 \mathrm{~mm}$, thickness $3 \mathrm{~mm}$.

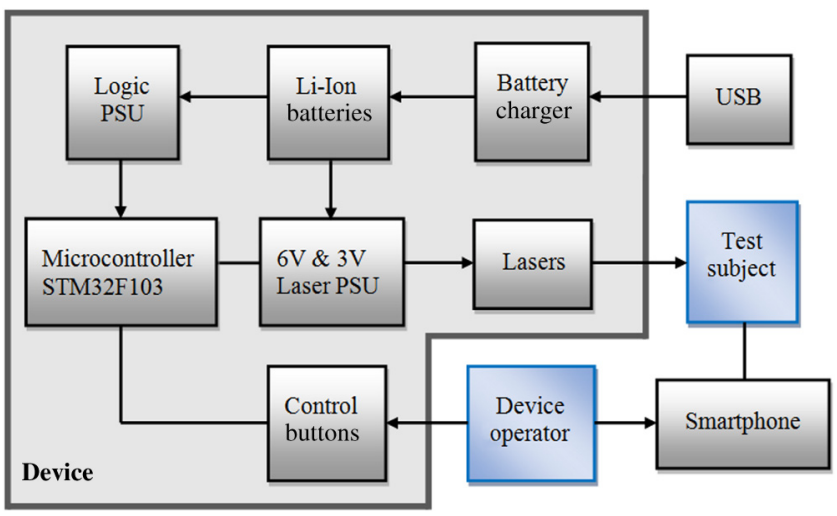

Fig. 5 Block diagram of the three-wavelength illumination unit, where PSU stands for power supply unit. with written consent of white skin (phototypes 1 and 2) patients, 22- to 64-years old. Nine vascular and pigmented skin lesions were examined, including three pigmented nevi, three seborrheic keratosis, and three hemangiomas; all mentioned skin malformations were diagnosed by an experienced dermatologist. The main goal of clinical measurements was to check if the above-described hardware and software can provide physiologically feasible information on already diagnosed skin malformations in terms of increased/decreased contents of the three regarded chromophores. Statistical quantitative analysis of skin malformations at this stage of development was not planned.

The patient measurement protocol included the following steps:

(1) Standard dermatology procedure to diagnose the skin pathology;

(2) Brief introduction to the patient about the new technology and the device;

(3) Written consent of patient to participate;

(4) Capture of calibration image of gray color reference under three-wavelength illumination;

(5) Adjustment of the device on the examined skin pathology and capturing its image under three-wavelength illumination;

(6) Repeated capture of the gray color reference image under three-wavelength illumination.

The recorded pathology images were further processed by PC using the above-described algorithm. Reference images taken before and after the pathology image were compared to make sure that illumination conditions during the procedure have not changed. Gray reference (instead of white) was selected to avoid overexposure of the image sensor. 
(a)

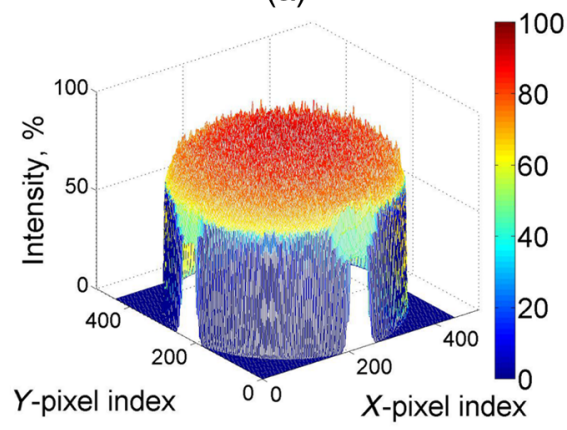

(d)

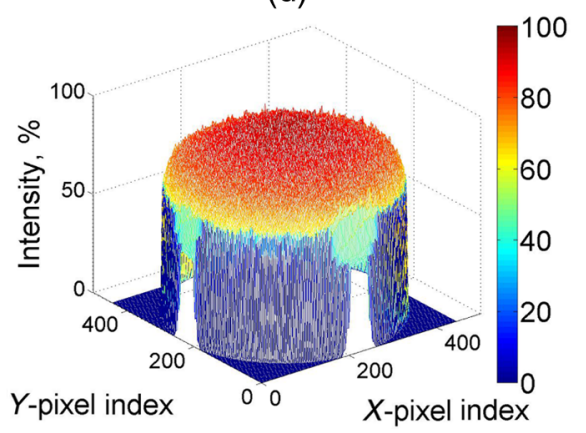

(b)

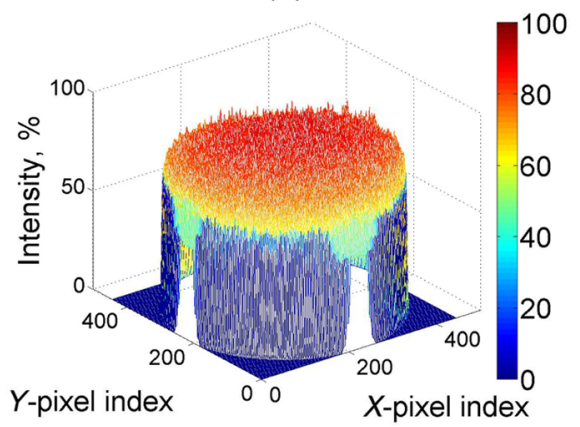

(e)

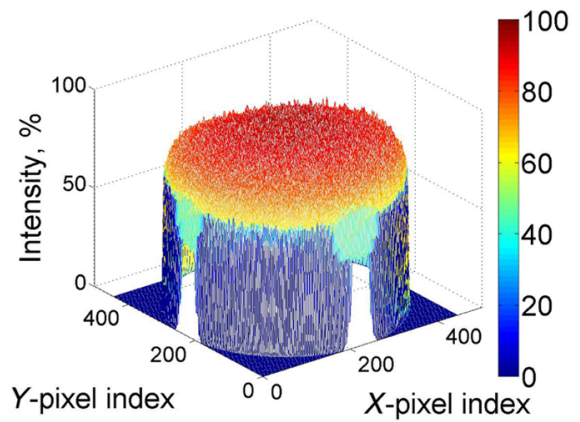

(c)

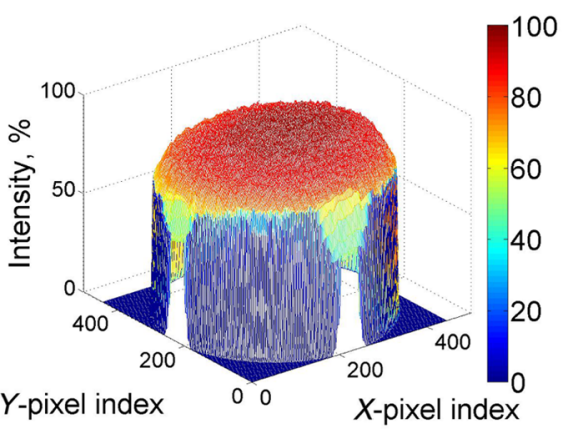

(f)

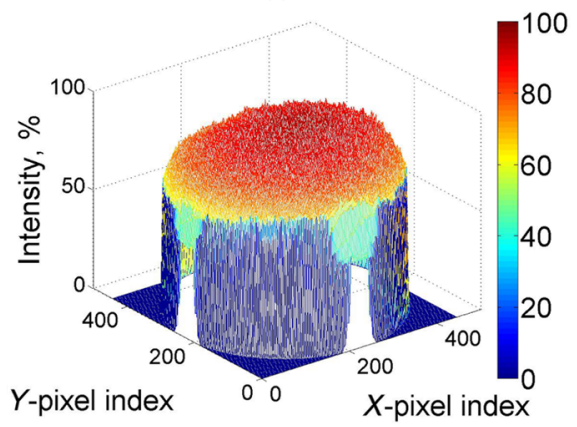

Fig. 6 Uniformity of white paper illumination at different illumination-detection combinations: (a) R-image at single-wavelength 659-nm illumination; (b) G-image at single-wavelength 532-nm illumination; (c) Bimage at single-wavelength 448-nm illumination; (d-f) R-, G-, and B-images at simultaneous three-wavelength illumination. Color scale represents relative intensities. Diameter of the illuminated area is $40 \mathrm{~mm}$.

\section{Results}

After processing the clinical images, skin chromophore maps were constructed and changes of malformation's chromophore content with respect to the adjacent healthy skin could be evaluated. Clinical models ${ }^{1}$ assume that the pigmented skin lesions represent an abnormal increase of epidermal melanin and the vascular lesions-increased supply of dermal arterial blood (rich of oxyhemoglobin) to the superficial layers of skin. As initially expected, we observed notable melanin content increase in all cases of both pigmented malformations: nevi and seborrheic keratosis. Increase of oxyhemoglobin content and decrease of deoxyhemoglobin were observed in all examined vascular malformations-hemangiomas. For illustration, images for these clinical cases related to each of the examined three pathologies are presented in Figs. 7-9.

The color photo of a malformation, taken under simultaneous three-wavelength illumination (a), is compared with the obtained concentration distribution maps of oxyhemoglobin (b), deoxyhemoglobin (c), and melanin (d). All pathology data are related to the adjacent healthy skin $(\Delta c=0)$ so that only increased and decreased concentrations are quantified by colors. Figure 7 shows how melanin content increases in nevi, without essential changes in the hemoglobin content. Similar responses were obtained from the other pigmented skin malformation, seborrheic keratosis (Fig. 8). Quite different chromophore composition changes were recorded for vascular hemangiomas (Fig. 9); melanin concentration in the pathology remained practically unchanged while the oxyhemoglobin content notably increased and the deoxyhemoglobin content decreased in comparison to healthy skin.
The color scales in Figs. 7-9 are calibrated in millimoles, so the increased or decreased chromophore content can be quantitatively analyzed and/or compared. It might help dermatologists to characterize the severity of pathology if clinical thresholds for particular chromophores in the malformation are established.

The grainy structure of chromophore maps at Figs. 7-9 is most probably caused by formation of laser speckles on the skin surface. This is a coherent effect and cannot be avoided even in scattered laser light.

\section{Discussion}

Generally, the obtained results have confirmed viability of the proposed approach for single-snapshot skin chromophore mapping with a smartphone. The compact add-on illuminator design [Figs. 3(a) and 4] ensured uniform skin illumination simultaneously at three-laser wavelengths (Fig. 6). The previously developed RGB monochromatic imaging concept, complemented by MC simulations of photon trajectories in skin, was successfully applied for mapping of pathologic concentration changes of skin melanin, oxyhemoglobin, and deoxyhemoglobin (Figs. 7-9). Results of this study may facilitate further developments of relatively simple and compact optical equipment for clinical diagnostics and monitoring; they also highlight the directions of further research, related to yet unsolved problems.

One of them is insufficient quality of chromophore maps due to laser speckles formed on the illuminated skin. In order to obtain speckle-free images, incoherent spectral line sources could be used. As an option, we assembled a three-wavelength illuminator based on high frequency electrodeless spectral lamps filled with an $\mathrm{Hg}-\mathrm{Cd}$ vapor mixture. However, such 
(a)
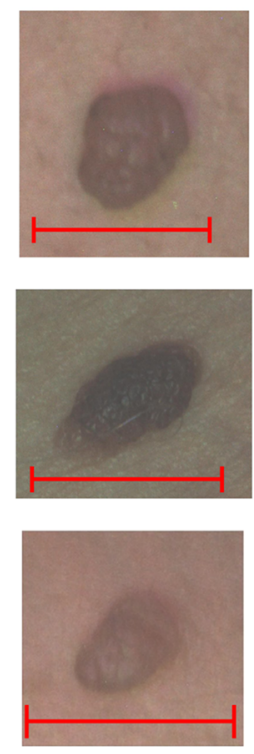

(b)
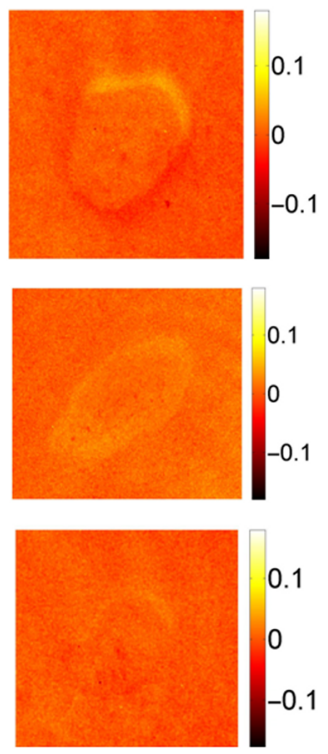

(c)
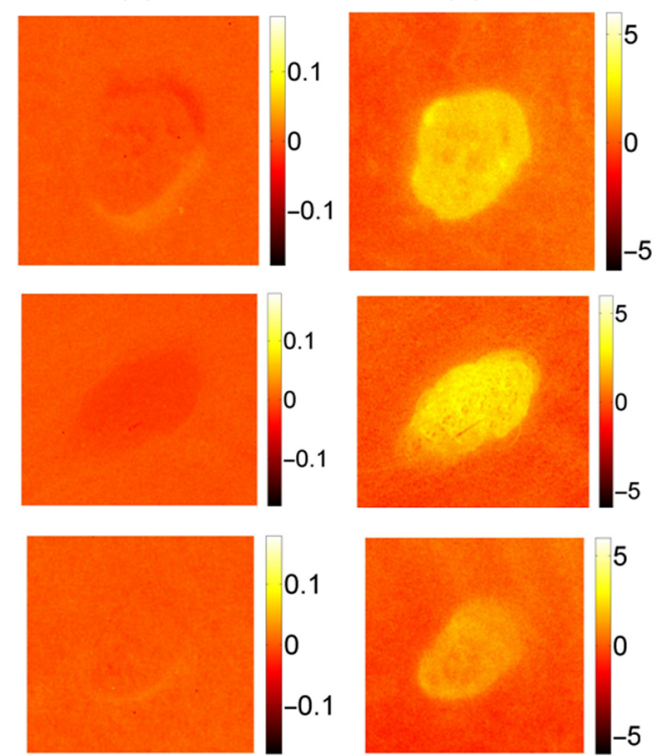

(d)

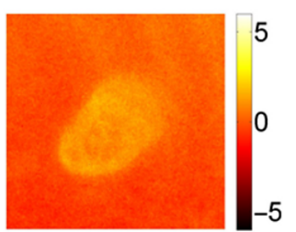

Fig. 7 (a) RGB image (scale bar: $5 \mathrm{~mm}$ ) and the corresponding maps representing chromophore concentration changes for three cases of pigmented nevi: (b) oxyhemoglobin, (c) deoxyhemoglobin, and (d) melanin. Units of the color scale: millimoles (mM).

(a)
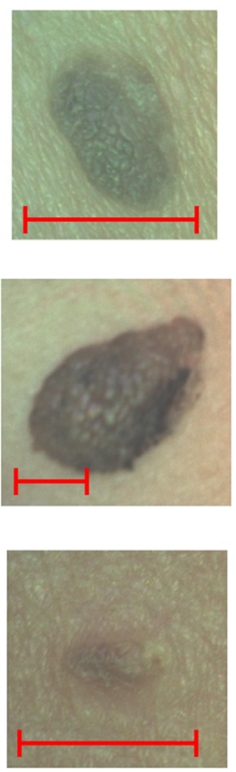

(b)
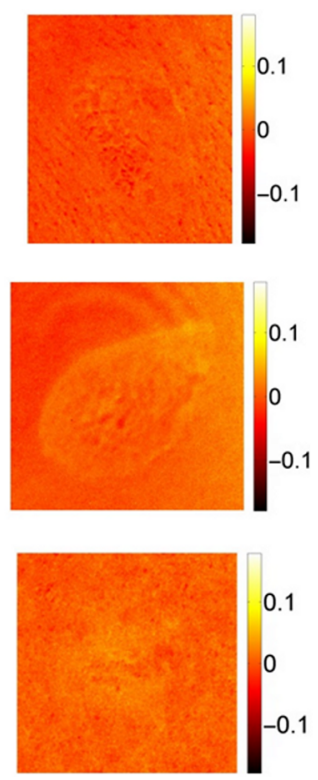

(c)
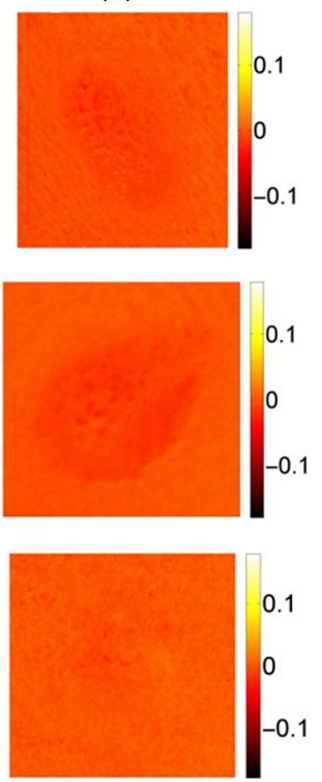

(d)
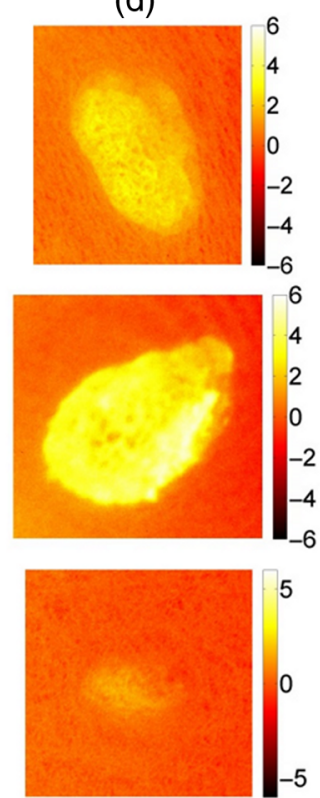

Fig. 8 (a) RGB image (scale bar: $5 \mathrm{~mm}$ ) and the corresponding maps representing chromophore concentration changes for three cases of pigmented seborrheic keratosis: (b) oxyhemoglobin, (c) deoxyhemoglobin, (d) melanin. Units of the color scale: millimoles (mM).

an illuminator required an additional high quality multibandpass filter which made the design too expensive, so probably more efforts are needed to develop other kinds of speckle-free multiwavelength illumination sources.

Another issue of further improvements is the skin absorption model; only three main skin chromophores were considered in this study, while bilirubin, $\beta$-carotene, lipids, and water may also contribute as skin absorbers. ${ }^{3,29}$ Therefore, image-processing algorithms based on more advanced skin models (e.g., 4chromophore or 5-chromophore) are to be developed in the future, along with further hardware developments. Smartphones comprise the conventional RGB image sensors, so only three output channels (R-, G-, and B-images) can be exploited; it limits to three the number of chromophores to be mapped by one snapshot. In this respect, a technology using several sets of simultaneous three-wavelength illumination should be developed in the future.

From the point of clinicians, the above-described technique would become useful for quantitative diagnostics only when a "gold standard" that sets the threshold (critical) values for 
(a)
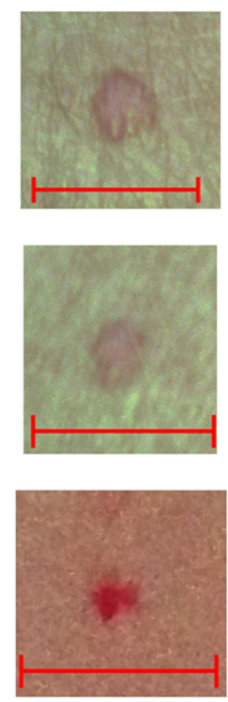

(b)
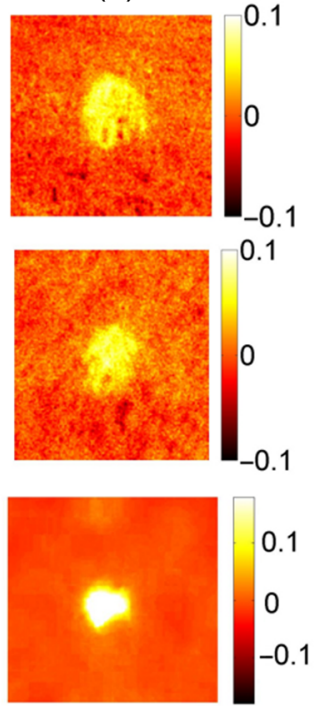

(c)
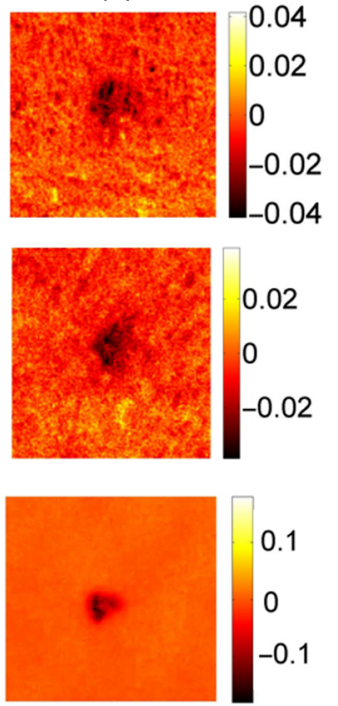

(d)
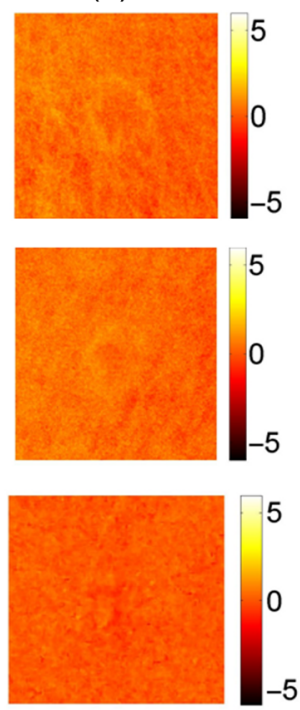

Fig. 9 (a) RGB image (scale bar: $5 \mathrm{~mm}$ ) and the corresponding maps representing chromophore concentration changes for three cases of vascular hemangioma: (b) oxyhemoglobin, (c) deoxyhemoglobin, (d) melanin. Units of the color scale: millimoles $(\mathrm{mM})$.

increased chromophore concentrations, related to particular pathologies, will be available. To achieve that, serious clinical trials with a much larger number of patients and statistical analysis of the results have to be carried out. Severity of skin pathology could be quantified also in terms of chromophore concentration ratio "pathology/healthy"; further research in this direction would be necessary as well. In addition, results of the image processing-chromophore distribution mapsshould appear on the screen of the used smartphone. Two approaches for that are to be examined: development of specific software application for particular (most popular) smartphone models or using a distant server that receives the captured images via mobile network and after processing sends back the calculated chromophore maps. The second option seems advantageous since it can be applied independently on the used smartphone model. As for potential hardware improvements, more handy for clinical use probably would be a separate multiwavelength illuminator which is first properly placed on the skin pathology and only then the smartphone attached for image capturing.

The mean path length in skin of the backscattered photons has been evaluated by means of MC simulations in this work. It would be useful to develop in the future techniques for direct determination of this parameter, e.g., using the photon timeof-flight measurement technique. ${ }^{41}$

\section{Conclusions}

This study confirmed the possibility to map three main skin chromophores by a single snapshot of a smartphone camera under combined spectral line illumination. Millions of professionals nowadays use smartphones, so the proposed relatively simple and inexpensive approach potentially may find real clinical and forensic applications. The main challenges of the future work will be: (i) to minimize or fully avoid laser speckle artifacts in spectral images of the illuminated skin area; (ii) to improve the imageprocessing algorithms and hardware details; (iii) to expand the range of chromophores that can be mapped, e.g., to add bilirubin which shows up in cases of skin bruises and postsurgery recovery.

\section{Disclosures}

No conflicts of interest, financial or otherwise, are declared by the authors.

\section{Acknowledgments}

This study was supported by the Latvian National Research Program SOPHIS under the Grant agreement \#10-4/VPP-4/ 11. Kind assistance of Dr. Aivars Vembris (ISSP, UL) in production of the light collecting/diffusing element is highly appreciated. $\mathrm{AB}$ acknowledges the support provided by Academy of Finland (Grant Nos. 296408 and 290596) and CSC-IT Center for Science, Finland, for computational resources. The fruitful collaboration between groups of two universities was facilitated by the European Commission COST action BM1205.

\section{References}

1. S. Chadwick, R. Heath, and M. Shah, "Abnormal pigmentation within cutaneous scars: a complication of wound healing," Indian J. Plast. Surg. 45(2), 403-411 (2012).

2. S. Cotton, "Astron Clinica SIAscope V," http://www.panchromos.com/ stories/astronclinica/ (1 February 2017).

3. R. R. Anderson and J. A. Parrish, "The optics of human skin," J. Invest. Dermatol. 77(1), 13-19 (1981).

4. P. Valisou et al., "New closed-form approximation for skin chromophore mapping," J. Biomed. Opt. 16(4), 046012 (2011).

5. E. C. Ruvolo et al., "Use of spectral imaging for documentation of skin parameters in face lift procedure," Proc. SPIE 7548, 75480A (2010).

6. D. N. Stratis et al., "Comparison of acousto-optic and liquid crystal tunable filters for laser-induced breakdown spectroscopy," Appl. Spectrosc. 55(8), 999-1004 (2001).

7. L. Randeberg, E. Larsen, and L. Svaasand, "Characterization of vascular structures and skin bruises using hyperspectral imaging, image analysis and diffusion theory," J. Biophoton. 3(1-2), 53-65 (2010).

8. D. Yudovsky et al., "Assessing diabetic foot ulcer development risk with hyperspectral tissue oximetry," J. Biomed. Opt. 16(2), 026009 (2011).

9. F. Vasefi et al., "Separating melanin from hemodynamics in nevi using multimode hyperspectral dermoscopy and spatial frequency domain spectroscopy," J. Biomed. Opt. 21(11), 114001 (2016). 
10. I. Kuzmina et al., "Contact and contactless diffuse reflectance spectroscopy: potential for recovery monitoring of vascular lesions after intense pulsed light treatment," J. Biomed. Opt. 16(4), 040505 (2011).

11. C. H. Morimoto and R. Chellappa, "Evaluation of image stabilization algorithms," IEEE Trans. Acoust. Speech Signal Process. 5, 2789-2792 (1988).

12. D. Jakovels and J. Spigulis, "RGB imaging system for mapping and monitoring of hemoglobin distribution in skin," Proc. SPIE 8158, 8158OR (2011).

13. D. Kapsokalyvas et al., "Spectral morphological analysis of skin lesions with a polarization multispectral dermoscope," Opt. Express 21(4), 4826-4840 (2013).

14. J. Spigulis and L. Elste, "Method and device for imaging of spectral reflectance at several wavelength bands," WO2013135311A1 (2012).

15. J. Spigulis and L. Elste, "Single snapshot RGB multispectral imaging at fixed wavelengths: proof of concept," Proc. SPIE 8937, 89370L (2014).

16. J. Spigulis, D. Jakovels, and L. Elste, "Towards single snapshot multispectral skin assessment," Proc. SPIE 8216, 82160L (2012).

17. J. Spigulis and I. Oshina, "Snapshot RGB mapping of skin melanin and hemoglobin," J. Biomed. Opt. 20(5), 050503 (2015).

18. Anonymous, "2 billion consumers worldwide to get smart(phones) by 2016," 2014, http://www.emarketer.com/Article/2-Billion-ConsumersWorldwide-Smartphones-by-2016/1011694 (30 November 2016).

19. A. C. Brewer et al., "Mobile applications in dermatology," J. Am. Med. Assoc. Dermatol. 149(11), 1300-1304 (2013).

20. N. MacKinnon et al., "Melanoma detection using smartphone and multimode hyperspectral imaging," Proc. SPIE 9711, 971117 (2016).

21. J. A. Wolf et al., "Diagnostic inaccuracy of smartphone applications for melanoma detection," J. Am. Med. Assoc. Dermatol. 149(4), 422-426 (2013).

22. S. Kim et al., "Smartphone-based multispectral imaging: system development and potential for mobile skin diagnosis," Biomed. Opt. Express 7(12), 5294 (2016).

23. I. Kuzmina et al., "Study of smartphone suitability for mapping of skin chromophores," J. Biomed. Opt. 20(9), 090503 (2015).

24. S. Prahl, "Tabulated molar extinction coefficient for hemoglobin in water," 1998, http://omlc.ogi.edu/spectra/hemoglobin/summary.html (30 November 2016).

25. T. Sarna and H. M. Swartz, "The physical properties of melanin," 1998, http://omlc.ogi.edu/spectra/melanin/eumelanin.html (30 November 2016).

26. D. Jakovels and J. Spigulis, "2-D mapping of skin chromophores in the spectral range 500-700 nm," J. Biophoton. 3(3), 125-129 (2010).

27. B. M. Tissue"Beer-Lambert law," 1996, http://elchem.kaist.ac.kr/vt/ chem-ed/spec/beerslaw.htm (30 November 2016).

28. D. Yudovsky and L. Pilon, "Retrieving skin properties from in vivo spectral reflectance measurements," J. Biophoton. 4(5), 305-314 (2011).

29. S. Jacques, "Optical properties of biological tissues: a review," Phys. Med. Biol. 58, R37-R61 (2013).

30. G. Petrov et al., "Human tissue color as viewed in high dynamic range optical spectral transmission measurements," Biomed. Opt. Express 3(9), 2154-2161 (2012).

31. S.-H. Tseng, A. Grant, and A. Durkin, "In vivo determination of skin near-infrared opticalproperties using diffuse optical spectroscopy," J. Biomed. Opt. 13(1), 014016 (2008).
32. M. Kirillin et al., "Simulation of optical coherence tomography images by Monte Carlo modeling based on polarization vector approach," $O p t$. Express 18(21), 21714-21724 (2010).

33. A. Bashkatov et al., "Optical properties of human skin, subcutaneous and mucous tissues in the wavelength range from 400 to $2000 \mathrm{~nm}$," J. Phys. D Appl. Phys. 38, 2543-2555 (2005).

34. A. Bykov, A. Doronin, and I. Meglinski, "Light propagation in turbid tissue-like scattering media," in Deep Tissue Imaging with Linear and Non-linear Optics, L. Shi and R. Alfano, Eds., Pan Stanford Publishing Pte Ltd., Singapore (2017).

35. A. V. Bykov, A. V. Priezzhev, and R. Myllylä, "Visualisation of structural inhomogeneities in strongly scattering media using the method of spatially-resolved reflectometry: Monte Carlo simulation," Quant. Electron. 41(6), 557-563 (2011).

36. L. P. Bass et al., "Parallel algorithm of the discrete ordinate method to laser impulse propagation simulation in turbid media," Nuovo Cimento C 33(1), 39-46 (2010).

37. J. Spigulis et al., "Method and device for smartphone mapping of tissue compounds," WO2017/012675A1, Riga, Latvia (2017).

38. T. Nomura, Sony LSI Design Incorporated, private correspondence.

39. A. Lihachev et al., "Autofluorescence imaging of basal cell carcinoma by smartphone RGB camera," J. Biomed. Opt. 20(12), 120502 (2015).

40. Anonymous, "High contrast linear polarizing film," 2017, https://www. edmundoptics.com/optics/polarizers/linear-polarizers/high-contrastlinear-polarizing-film/3435/ (23 February 2017).

41. A. Dzerve et al., "Time of flight for photons in human skin," J. Biomed. Photonics Eng. 2(3), 030301 (2016).

Janis Spigulis is the head of Biophotonics Laboratory at the Institute of Atomic Physics and Spectroscopy (IAPS) and professor at the Physics Department, University of Latvia. His research is focused on optical noncontact human skin diagnostics and monitoring.

Ilze Oshina is a research assistant at Biophotonics Laboratory of IAPS and a postgraduate student at the Faculty of Physics and Mathematics, University of Latvia. Her research interests are related to in vivo skin imaging and chromophore mapping by advanced photonic technologies. She had participated in several research projects related to skin diagnostics.

Anna Berzina is a research assistant at Biophotonics Laboratory of IAPS and certified dermatologist, expert in noninvasive diagnostics of skin and its treatment with lasers and other light-emitting devices. She has taken part in research projects related to optical skin diagnostics and recovery monitoring and is coauthor of more than 10 scientific publications.

Alexander Bykov received his MSc degree in 2005 and his $\mathrm{PhD}$ in 2008 from Lomonosov Moscow State University, Russia. He received his DSc (Tech.) degree from the Faculty of Technology, University of Oulu, Finland, in 2010. Currently, he is a senior researcher in the Optoelectronics and Measurement Techniques Laboratory, University of Oulu, Finland. 